\title{
Role of methyltransferase-like enzyme 3 and methyltransferase-like enzyme 14 in urological cancers
}

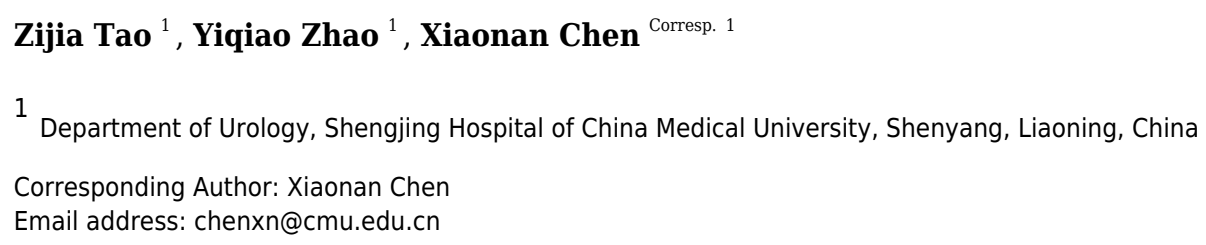

N6-methyladenosine (m6A) modifications can be found in eukaryotic messenger RNA (mRNA), long non-coding RNA (IncRNA), and microRNA (miRNA). Several studies have demonstrated a close relationship between m6A modifications and cancer cells. Methyltransferase-like enzyme 3 (METTL3) and methyltransferase-like enzyme 14 (METTL14) are two major enzymes involved in m6A modifications that play vital roles in various cancers. However, the roles and regulatory mechanisms of METTL3 and METTL14 in urological cancers are largely unknown. In this review, we summarize the current research results for METTL3 and METTL14 and identify potential pathways involving these enzymes in kidney, bladder, prostate, and testicular cancer. We found that METTL3 and METTL14 have different expression patterns in four types of urological cancers. METTL3 is highly expressed in bladder and prostate cancer and plays an oncogenic role on cancer cells; however, its expression and role are opposite in kidney cancer. METTL14 is expressed at low levels in kidney and bladder cancer, where it has a tumor suppressive role. Low METTL3 or METTL14 expression in cancer cells negatively regulates cell growthrelated pathways (e.g., mTOR, EMT, and P2XR6) but positively regulates cell death-related pathways (e.g., P53, PTEN, and Notch1). When METTL3 is highly expressed, it positively regulates the NF-kB and SHH-GL1pathways but negatively regulates PTEN. These results suggest that although METTL3 and METTL14 have different expression levels and regulatory mechanisms in urological cancers, they control cancer cell fate via cell growthand cell death-related pathways. These findings suggest that m6A modification may be a potential new therapeutic target in urological cancer. 
1 Role of methyltransferase-like enzyme 3 and

2 methyltransferase-like enzyme 14 in urological

3 cancers

4

5

6

7 Zijia Tao ${ }^{1}$, Yiqiao Zhao ${ }^{1}$, Xiaonan Chen ${ }^{1, *}$

8

$9{ }^{1}$ Department of Urology, Shengjing Hospital of China Medical University, Shenyang, Liaoning

10 110004, People's Republic of China

11

12

13

* Corresponding Author:

14 Xiaonan Chen

Department of Urology, Shengjing Hospital of China Medical University, No. 36 Sanhao Street, Heping District, Shenyang 110004, Liaoning, People's Republic of China

17 Email: chenxn@cmu.edu.cn

18

19

20

21

22

23

24

25

Peer] reviewing PDF | (2020:03:46959:2:0:NEW 26 Jun 2020) 


\section{Abstract}

28

29

30

31

32

33

34

35

36

37

38

39

40

41

42

43

44

45

46

47

48

49

50

51

52

53

54

55

56

57

58

59

N6-methyladenosine (m6A) modifications can be found in eukaryotic messenger RNA (mRNA), long non-coding RNA (lncRNA), and microRNA (miRNA). Several studies have demonstrated a close relationship between m6A modifications and cancer cells. Methyltransferase-like enzyme 3 (METTL3) and methyltransferase-like enzyme 14 (METTL14) are two major enzymes involved in m6A modifications that play vital roles in various cancers. However, the roles and regulatory mechanisms of METTL3 and METTL14 in urological cancers are largely unknown. In this review, we summarize the current research results for METTL3 and METTL14 and identify potential pathways involving these enzymes in kidney, bladder, prostate, and testicular cancer. We found that METTL3 and METTL14 have different expression patterns in four types of urological cancers. METTL3 is highly expressed in bladder and prostate cancer and plays an oncogenic role on cancer cells; however, its expression and role are opposite in kidney cancer. METTL14 is expressed at low levels in kidney and bladder cancer, where it has a tumor suppressive role. Low METTL3 or METTL14 expression in cancer cells negatively regulates cell growth-related pathways (e.g., mTOR, EMT, and P2XR6) but positively regulates cell death-related pathways (e.g., P53, PTEN, and Notch1). When METTL3 is highly expressed, it positively regulates the NF-kB and SHH-GL1pathways but negatively regulates PTEN. These results suggest that although METTL3 and METTL14 have different expression levels and regulatory mechanisms in urological cancers, they control cancer cell fate via cell growth- and cell death-related pathways. These findings suggest that m6A modification may be a potential new therapeutic target in urological cancer.

\section{Introduction}

Chemical modifications of eukaryotic RNA have been known for decades. However, the roles of these modifications in tumor development were largely unknown until recent years. According to the data of MODOMICS, a database of RNA modification pathways, 163 different RNA chemical modifications have been identified in all living organisms (Boccaletto et al. 2018). The N6-methyladenosine (m6A) modification is one of the most common, invertible, and abundant modifications found on eukaryotic mRNA, miRNA, lncRNAs, and other RNA molecules. These modifications affect the transcription, processing, translation, and metabolism of these RNA molecules (Zheng et al. 2019). The m6A modification occurs by a dynamic process involving three major classes of enzymes: 'Writers,' 'Erasers,' and 'Readers' (Vu et al. 2019). Writers include methyltransferase-like enzyme 3 (METTL3), methyltransferase-like enzyme 
60 (METTL14), Wilms tumor 1-associated protein (WTAP), RNA binding motif protein 15/15B 61 (RBM15/15B), and vir-like M6A methyltransferase-associated (VIRMA), which catalyze the 62 generation of m6A. Erasers, which include fat and obesity-related protein (FTO) and alkB 63 homolog 5 (ALKBH5), are responsible for demethylation. Readers recognize the m6A 64 methylation and generate functional signals (Chen et al. 2019b). This latter class of enzymes includes eukaryotic initiation factor (eIF3), the IGF2 mRNA binding protein (IGF2BP) family,

66 67 68 69 70 71 72 73 74 75 76 77 78 79 80 the heterogeneous nuclear ribonucleoproteins (HNRNP) protein family, and proteins that contain a YT521-B homology (YTH) domain.

METTL3 is a 70-kDa protein that was first identified in Hela cell lysates (Bokar et al. 1997). It contains two domains that bind S-adenosylmethionine (SAM) and catalyze the formation of m6A in RNA (Leach \& Tuck 2001). WTAP promotes METTL3 localization to nuclear spots and greatly improves its catalytic activity (Ping et al. 2014). Studies have also shown that METTL3 acts as a positive regulator of mRNA translation independent of methyltransferase activity: promoting translation by involving in translation initiation mechanisms in the cytoplasm (Ke et al. 2017). It has been reported that METTL3 can play the role without METTL14 and can promote translation of specific mRNAs independently of its catalytic activity in vitro (Ke et al. 2017). METTL3 is the core catalytic activity in the N6-methyltransferase complex formed by the METTL3-METTL14 heterodimer. Adenosine residues at the N (6) position of some RNAs are methylated by this complex (Alarcon et al. 2015a; Alarcon et al. 2015b; Bokar et al. 1997; Dominissini et al. 2012; Du et al. 2018; Liu et al. 2015; Meyer et al. 2015; Scholler et al. 2018; Sledz \& Jinek 2016; Wang et al. 2016a; Wang et al. 2016b; Wang et al. 2014; Xiang et al. 2017; Zhong et al. 2018). METTL14 is a scaffold for bound RNA and identifies the substrate of the N6-methyltransferase complex formed by the METTL3-METTL14 heterodimer (Liu et al. 2014; Liu et al. 2015; Ping et al. 2014; Scholler et al. 2018; Sledz \& Jinek 2016; Wang et al. 2016a; Wang et al. 2016b). METTL14 shares about 22\% sequence identity and nearly identical topology with domains found in METTL3. When part of the METTL3-METTL14 heterodimer, METTL14 is thought to assume a pseudo-methyltransferase function that helps bind RNA and stabilize METTL3. However, it is possible that methyltransferase activity mediated by METTL14 may occur after the binding of additional factors (Wang et al. 2017). In mRNA, the methylation site is located in the 5'-[AG] GAC-3' consensus site found in some mRNAs, which plays an important role in mRNA stability, processing, translation efficiency, and editing (Alarcon et al. 2015a; Alarcon et al. 2015b; Bokar et al. 1997; Dominissini et al. 2012; Liu et al. 2015; Meyer et al. 2015; Wang et al. 2014; Xiang et al. 2017). Methylation is completed after the mRNA is released into the nucleoplasm and promotes mRNA instability and degradation (Ke et al. 2017). 
95

96

97

98

99

100

101

102

103

104

105

106

107

108

109

110

111

112

113

114

115

116

117

118

119

120

121

122

123

124

125

126

127

In recent years, the role of m6A in various cancers, including leukemia, brain, cervical, endometrial, breast, liver, and lung cancer, has been revealed (Chen et al. 2018; Choe et al. 2018; Liu et al. 2018; Vu et al. 2017; Weng et al. 2018; Zhang et al. 2016; Zhang et al. 2017). m6A serves a regulatory function in oncogenesis and development by modifying many target genes (Deng et al. 2018; Liu et al. 2018). Interestingly, m6A may have oncogenic or suppressive functions depending on the cellular environment (Cui et al. 2017; Li et al. 2017b; Lin et al. 2016; Ma et al. 2017; Visvanathan et al. 2018; Vu et al. 2017; Zhang et al. 2016; Zhang et al. 2017). METTL3 mediates YTHDF2-dependent post-transcriptional silencing of SOCS2(Suppressor of Cytokine Signaling 2) to promote liver cancer progression (Chen et al. 2018). METTL14 is expressed at low levels in liver cancer and hematopoietic stem cells, and it impairs acute myelocytic leukemia (AML) oncogenesis (Weng et al. 2018). METTL14 can also inhibit liver oncogenesis and metastasis (Li et al. 2017b). Some studies have shown that abnormal m6A modification is necessary for oncogenesis and progression (Cui et al. 2017; Li et al. 2017b; Lin et al. 2016; Ma et al. 2017; Visvanathan et al. 2018; Vu et al. 2017; Zhang et al. 2016; Zhang et al. 2017), suggesting that the pathway involved in the m6A modification may be a promising therapeutic target in oncology.

Urological tumors include kidney, bladder, prostate, and testicular cancer. After decades of research, there have been significant improvements in the treatment of these cancer types; however, drug resistance and low survival rates still prevail. In addition, the lack of accurate and useful molecular markers for timely diagnosis and prognosis assessment of patients has led to unsatisfactory treatment results (Cai et al. 2019; Cheng et al. 2018; Cheng et al. 2019; Gong et al. 2019). In this review, we summarize the present research progress in understanding the roles of METTL3 and METTL14 in urological tumors and their potential as treatment and diagnostic markers.

\section{Survey methodology}

In order to search literatures exhaustively, we used keywords 'METTL3', 'Methyltransferaselike enzyme 3', 'METTL14', 'Methyltransferase-like enzyme 14', 'kidney cancer', 'renal cell carcinoma', 'bladder cancer', 'prostate cancer', and 'testicular cancer' to search articles in the PubMed, Web of Science and CNKI. We excluded the articles which were not associated with METTL3, METTL14 and urological cancers.

\section{Methyltransferase in urological cancers}




\section{1.Kidney cancer}

130 According to the GLOBOCAN (Global Cancer Observatory) statistics, 403,262 people were 131 diagnosed with renal cancer throughout the world, and 175,098 people died in 2018, making 132 renal cancer the 14th most common cancer in the world. There is a higher incidence of this 133 cancer in males than in females (Bray et al. 2018). Many patients remain asymptomatic until 134 renal masses progress to an advanced stage because of the position of the kidneys in the body. 135 Based on the World Health Organization (WHO) 2016 classification, renal cell carcinoma(RCC) 136 are divided into three main subcategories: 1) clear cell renal cell carcinoma (ccRCC), the most 137 common and aggressive type; 2) chromophobe renal cell carcinoma (chRCC); 3) papillary renal 138 cell carcinoma (pRCC), which consists of types 1 and 2 (Hao et al. 2019; Moch et al. 2016). Analysis of methyltransferases in kidney cancer, including ccRCC, indicates that both METTL3 and METTL14 are tumor suppressors in this disease (Gong et al. 2019; Li et al. 2017a; Wang et al. 2019; Zhou et al. 2019).

142

\subsection{METTL3 in kidney cancer}

144

145

146

147

148

149

150

151

152

153

154

155

156

157

158

159

160

METTL3 is more prone to copy number variations (CNV) or mutation than other genes in ccRCC, and patients affected by METTL3 shallow deletions (a form of CNV) have poorer disease-free survival (DFS) and overall survival (OS) (Zhou et al. 2019). METTL3 mRNA and protein expression are low in RCC. Its expression level is negatively related to higher histological grade, larger tumor size, shorter OS, and shorter DFS (Li et al. 2017a; Zhou et al. 2019). Knocking down of METTL3 expression in RCC cell lines significantly increases proliferation, migration, and invasion (Li et al. 2017a). The VHL-HIF-ZNF217(Von HippelLindau- Hypoxia Inducible Factor- Zinc Finger Protein 217)-METTL3 pathway may be involved in $\mathrm{m} 6 \mathrm{~A}$ regulation in ccRCC cells by mediating two downstream m6A targets, the $\mathrm{PI} 3 \mathrm{~K} / \mathrm{AKT} / \mathrm{mTOR}$ (Phosphatidylinositol 3-Kinase/AKT, also known as Protein Kinase B/mammalian Target of Rapamycin) and p53 signaling pathways (Li et al. 2017a; Zhou et al. 2019). The PI3K/AKT/mTOR pathway plays a significant role in cell proliferation, growth, and survival (O'Reilly et al. 2006; Shaw \& Cantley 2006). In addition, METTL3 may inhibit the invasion and migration of RCC through the epithelial-mesenchymal transition (EMT) pathway (Li et al. 2017a).

GSEA analysis of ccRCC patient tumors suggests that low METTL3 expression levels may be related to some critical biological processes, such as the mTOR pathway, adipogenesis, and 
161

162

163

164

165

166

167

168

169

170

171

172

173

174

175

176

177

178

179

180

181

182

183

184

185

186

187

188

189

190

191

192

193

reactive oxygen species (ROS), which partially validates the RCC cell line results. Based on these data, the mTOR pathway may be the key target of the m6A modification in kidney cancer. Alternatively, METTL3 also regulates the cell cycle. Downregulation of METTL3 significantly decreases G1 cell cycle arrest, whereas the upregulation of METTL3 increases G1 arrest (Zhou et al. 2019).

\subsection{METTL14 in kidney cancer}

Like METTL3, patients with kidney cancer are more predisposed to METTL14 mutations or CNV, and patients affected by shallow deletions of METTL14 have a poorer OS and DFS (Zhou et al. 2019). METTL14 is mainly located in the nucleus of ccRCC cells. Compared with normal kidney tissues, METTL14 mRNA expression is significantly lower in ccRCC tumors. METTL14 expression levels are negatively correlated with RCC pathological and clinical stages, and positively correlated with OS (Wang et al. 2019). As with METTL3, the VHL-HIF-ZNF217METTL14 pathway regulates m6A in ccRCC cells via the PI3K/AKT/mTOR and p53 signaling pathways (Li et al. 2017a; Zhou et al. 2019). METTL14 has also been associated with two other regulating pathways, including P2RX6(Purinergic Receptor P2X 6) and PTEN (Phosphatase and Tensin Homolog) (Gong et al. 2019; Wang et al. 2019). P2RX6 is a non-selective cation channel protein that is a preferred receptor for ATP (Chadet et al. 2014; North 2002). METTL14 expression is negatively correlated with P2RX6. Low METTL14 expression is associated with a shorter OS, while low P2RX6 expression correlates with a longer OS. METTL14 may increase the pre-mRNA splicing of P2RX6 by increasing the methylation of P2RX6 mRNA, thereby inhibiting P2RX6. Low METTL14 expression in cancer cells leads to high P2RX6 expression via the ATP-P2RX6-Ca2+-p-ERK1/2(Extracellular Regulated protein Kinases 1/2)MMP9(Matrix Metallopeptidase 9) signaling pathway, which promotes renal tumor cell metastasis and invasion (Gong et al. 2019). PTEN is a tumor suppressor, whose duty is to encode phosphatidylinositol-3,4,5-trisphosphate 3-phosphatase to preferentially dephosphorylate phosphoinositide substrates. The METTL14 mRNA expression level is positively associated with PTEN. Patients with low METTL14 and PTEN expression levels have a shorter OS. METTL14 stabilizes PTEN mRNA by regulating the m6A levels on the PTEN mRNA. PTEN acts as a tumor suppressor by negatively regulating the AKT/PKB signaling pathway.

Synergistic effects may occur through the interaction of EIF3A (Eukaryotic Translation Initiation Factor 3 Subunit A) and METTL14, which regulates kidney cancer progression. In addition, 24 circRNAs (e.g., circ-0023414 and circ-0031772) interact with four miRNAs (miR-130a-3p, miR- 
194

195

196

197

198

199

200

201

202

203

204

205

206

207

208

209

210

211

212

213

214

215

216

217

218

219

220

221

222

223

224

225

106b-5p, miR-130b-3p, and miR-301a-3p), which have a negative relationship with METTL14 mRNA (Wang et al. 2019). These circRNAs may act as miRNA sponges to regulate METTL14 mRNA (Fig.1).

\section{Bladder Cancer}

Bladder cancer was the 12th most common cancer globally in 2018, with 549,393 newly diagnosed cases and 199,922 deaths. The incidence of bladder cancer varies by gender, with males at higher risk (Bray et al. 2018). Urothelial carcinoma is a common histological type of bladder cancer. Non-papillary muscle-invasive and papillary non-muscle-invasive tumors are the two main types of this disease (Sanli et al. 2017; Wu et al. 2019). Most of the available research on methyltransferases in bladder cancer suggests that METTL3 is a oncogene, whereas METTL14 is a tumor suppressor.

\subsection{METTL3 in bladder cancer}

METTL3 is highly expressed in bladder cancer tissues (Chen et al. 2019a). Overexpression of METTL3 significantly promotes the growth and invasion of bladder tumor cells. In contrast, METTL3 knockdown abrogates the proliferation, invasion, and viability of bladder cancer cells and reduces the proportion of cells in the $\mathrm{S}$ phase of the cell cycle while increasing the proportion in G1. METTL3 may maintain the characteristics of bladder cancer stem cells by inducing the m6A modification of SOX2(SRY-Box Transcription Factor 2), a marker of bladder cancer stem cells both in vivo and in vitro (Zhu et al. 2017). Patients with high METTL3 expression in bladder cancer have higher histological scores, worse prognosis, and shorter survival time. Thus, METTL3 exhibits a oncogenic role in bladder cancer.

The AFF4/NF-кB/MYC (AF4/FMR2 Family Member 4/ Nuclear Factor Kappa B/

Myelocytomatosis oncogene) signaling network plays a vital role in the upregulation of METTL3 in bladder cancer. METTL3 can directly increase the abundance of m6A sites on the MYC mRNA to improve the stability of MYC transcripts and increase MYC protein expression. It has a similar effect on AFF4 mRNA and protein. AFF4 protein directly binds to the MYC promoter to extend MYC transcription and upregulate MYC expression. METTL3 may also promote the expression of IKBKB (Inhibitor of Nuclear Factor Kappa B Kinase Subunit Beta) and RELA (V-Rel Reticuloendotheliosis Viral Oncogene Homolog A), which are two key

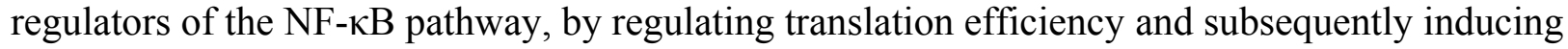


226

227

228

229

230

231

232

233

234

235

236

237

238

239

240

241

242

243

244

245

246

247

248

249

250

251

252

253

254

255

256

257

258

259

MYC transcription. Thus, m6A modifications mediated by METTL3 through different signaling pathways converge at MYC expression. This m6A-regulated malignant regulatory network effectively increases MYC protein levels in bladder cancer and may lead to difficulties in reducing MYC by blocking a single signaling pathway (Cheng et al. 2019). The METTL3-DGCR8(DiGeorge Syndrome Critical Region 8)-pri-mi221/222-PTEN pathway also mediates the upregulation of METTL3 in bladder cancer. METTL3 can actively regulate pri-miR221/222 in an m6A-dependent manner by interacting with DGCR8, a micro-processor protein that promotes the processing of pri-miR221/222 into mature miR221/222 in bladder cancer. miR221/222 binds to the 3'-untranslated region (UTR) of PTEN mRNA, leading to decreased PTEN mRNA and protein expression (Han et al. 2019).

Three other pathways are also associated with METTL3 upregulation in bladder cancer, including the METTL3 -CDCP1(CUB Domain Containing Protein 1), METTL3-ITGA6(Integrin Subunit Alpha 6), and METTL3/YTHDF2-SETD7/KLF4(SET Domain Containing Lysine Methyltransferase 7/ Kruppel Like Factor 4) m6A axes. METTL3 and CDCP1 are both upregulated in bladder cancer patient samples and related to bladder cancer progression. Inhibition of the METTL3-CDCP1 axis reduces the growth and progression of bladder cancer cells and chemical-transformed cells. The METTL3-CDCP1 axis and chemical carcinogens have synergistic effects on the malignant transformation of uroepithelial cells and bladder cancer oncogenesis (Yang et al. 2019). In the METTL3-ITGA6 axis, METTL3 highly enriches the m6A methylation levels in the ITGA6 mRNA 3'-UTR region, which promotes the translation of ITGA6 mRNA. The binding of YTHDF1/YTHDF3 to the m6A motif in the ITGA6 3'-UTR region further increases ITGA6 translation. This overexpression of ITGA6 increases the adhesion, proliferation, and migration of bladder tumor cells and enhances their metastasis. Therefore, ITGA6 is a crucial target of METTL3 function in bladder cancer (Jin et al. 2019). METTL3 also catalyzes m6A modifications in the mRNAs of SETD7 and KLF4, two tumor suppressors that are part of the METTL3/YTHDF2-SETD7/KLF4 m6A axis. YTHDF2 recognizes these m6A modifications and degrades the SETD7 and KLF4 mRNAs, leading to bladder cancer progression (Xie et al. 2020).

Although most studies suggest that METTL3 can foster bladder cancer growth and progression, one study suggests that METTL3 can act as a bladder tumor suppressor. Zhao et al. (2019) identified METTL3 as a driver gene in a bladder cancer cohort using the integrated statistical model-based method called driver MAPS. However, in the subsequent experimental verification of this finding, the researchers found that METTL3 knockdown significantly increased cell proliferation. Furthermore, METTL3 somatic mutations could promote cancer cell growth by 
260

261

262

263

264

265

266

267

268

269

270

271

272

273

274

275

276

277

278

279

280

281

282

283

284

285

286

287

288

289

290

291

292

interrupting RNA methylation. Therefore, they believe that METTL3 acts as a tumor suppressor for bladder cancer (Zhao et al. 2019).

\subsection{METTL14 in bladder cancer}

METTL14 is expressed at low levels in bladder cancer and bladder tumor-initiating cells (TICs). Bladder cancer TICs possess self-renewal, differentiation, and tumor-initiating properties. METTL14 inhibits these properties along with the maintenance and metastasis of bladder TICs. METTL14 expression is negatively associated with the severity of bladder cancer and clinical outcome. METTL14 is significantly related to the T stage of the TNM stage system (Chen et al. 2019a).

Notch1 plays an important part in bladder oncogenesis and TICs self-renewing. It is a downstream target of METTL14. m6A modification of Notch1(Neurogenic Locus Notch Homolog Protein 1) decreases its RNA stability, leading to inhibition of bladder cancer and bladder tumor-initiating cells (Gu et al. 2019). Thus, METTL14 is a tumor suppressor in bladder cancer, acting through the METTL14-Notch1 pathway (Fig.2).

\section{Prostate Cancer}

Prostate cancer was the third most common cancer worldwide in 2018, with 1,276,106 newly diagnosed cases and 358,989 deaths (Bray et al. 2018). Because of the aging of the growing population, prostate cancer has become a main public health problem for men (Center et al. 2012). This tumor is often silent in clinical practice and usually found after it invades other tissues (Guo et al. 2019; Roobol \& Carlsson 2013; Shen \& Abate-Shen 2010). Studies of METTL3 in prostate cancer suggest that it is a oncogene.

METTL3 protein and mRNA expression levels in prostate cancer are significantly higher than those in adjacent benign tissue. METTL3 is mainly localized to the nucleus of prostate cells, with a small amount in the cytoplasm. METTL3 mRNA and protein levels are positively correlated with prostate-specific antigen (PSA) values and Gleason scores. Therefore, METTL3 plays an oncogenic role in prostate cancer and may be used in combination with PSA as a diagnostic marker for this disease (Xianyong et al. 2019).

Knockdown of METTL3 in prostate cancer cell lines reduces the m6A content and inhibits survival, cell proliferation, colony formation, and invasion. Mechanistic analysis indicated that there is decreased GLI1(Glioma-Associated Oncogene Family Zinc Finger 1) expression after METTL3 depletion. GLI1 is an important component of the SHH (Sonic Hedgehog Signaling 
293 Molecule)-GLI signaling pathway that is positively correlated with prostate cancer severity. 294 GLI1 is a negative modulator of the androgen receptor and contributes to the androgen295 independent growth of prostate cancer. c-Myc and cyclin D1 mRNA levels (SHH signaling 296 downstream targets) are also inhibited, resulting in apoptosis (Cai et al. 2019).

297 METTL3 expression is higher in prostate cancer than in normal prostate tissue, especially in 298 prostate cancer with bone metastasis ( $\mathrm{Li}$ et al. 2020). High METTL3 expression is positively 299 300 301 302 303 304 correlated with prostate cancer progression and poor prognosis. METTL3 overexpression increases the m6A levels of integrin $\beta 1$ (ITGB1) mRNA. HuR (also known as ELAV Like RNA Binding Protein 1) interacts with this modified mRNA to increase its stability and promote protein expression, making prostate cancer cells capable of adhering to collagen I in bone marrow stroma. This finding can explain the mechanism of prostate cancer bone metastasis to some extent. METTL3 also increases the m6A methylation of lymphoid enhancer-binding factor 1 (LEF1) mRNA, which promotes its protein expression and the progression of prostate cancer by activating the Wnt- $\beta$-catenin pathway (Ma et al. 2020). Thus, METTL3 is involved in the regulation of multiple pathways and mechanisms in prostate cancer and may have a pivotal position in this complex regulatory network. Although there are not many reports on $\mathrm{m} 6 \mathrm{~A}$ in prostate cancer, existing articles describe the mechanisms and research prospects of 'Writer' enzymes. Higher VIRMA expression levels are detected in metastatic castration-resistant prostate cancer (mCRPC) cells. Patients with high VIRMA expression have a significantly shorter disease-free survival. METTL3, METTL14, WTAP, and VIRMA form a methyltransferase complex (MTC); however, each component can function independently in other cellular processes. The knockout of VIRMA triggers a compensatory feedback loop that enhances the expression of the catalytic METTL3 subunit. However, compensatory METTL3 overexpression is insufficient to maintain MTC function without VIRMA (Barros-Silva et al. 2020) (Fig.3).

\section{Testicular Cancer}

320

321

322

323

324

325 326
According to the GLOBOCAN statistics, 71,105 people were diagnosed with testicular cancer globally in 2018, and 9,507 people died (Bray et al. 2018). More than $95 \%$ of testicular neoplasms are testicular germ cell tumors (TGCTs), which form two subclasses: germ-cell neoplasia in situ (GCNIS)-related and GCNIS-unrelated tumors. The GCNIS-related tumors include seminomas (SEs) and non-seminoma tumors (NSTs) (Moch et al. 2016). Although the morbidity and mortality of testicular tumors are not high compared to other urological tumors, there are no accurate and effective biomarkers for treatment (Lobo et al. 2018). There have been 
327 few studies on METTL3 and METTL14 in testicular cancer. In testicular germ cell tumor cell 328 lines and tissues, METTL3 appears to be the main 'Writer' enzyme. METTL14 expression can 329 be detected but only at moderate levels. Its expression in SEs is significantly higher than in 330 embryonal carcinoma (Nettersheim et al. 2019). METTL14 is expressed at lower levels in SEs

331

332

333

334

335

336

337 338

\section{9}

340

341

342

343

344

345

346

347

348

349

350

351

352

353

354

355

356

357

358

359

360

361 compared to NSTs (Lobo et al. 2018). In contrast, the mRNA expression levels of other m6Arelated enzymes (e.g., VIRMA and YTHDF3) in SEs are higher than in NSTs. VIRMA expression is positively correlated with YTHDF3 expression levels. These results suggest that m6A enzymes mainly contribute to the SE phenotype, but not other subtypes. Because of the expression of VIRMA and YTHDF3 in SEs, both enzymes may represent new candidate biomarkers for SE patient management (Lobo et al. 2019). So far, we believe that m6A may be a new direction to break through the current dilemma of testicular cancer.

\section{Conclusions}

From the limited studies available, we found that METTL3 and METTL14 have different expression patterns in four types of urological cancer (kidney, bladder, prostate, and testicular cancer). METTL3 is highly expressed in bladder and prostate cancer, where it plays oncogenic role. In contrast, METTL3 expression is low in kidney cancer. METTL14 is expressed at low levels in kidney and bladder cancer playing tumor suppressive role. High METTL14 expression has not been found in urological cancers. Regardless of the type of urological cancer, low METTL3 or METTL14 expression negatively regulates cell growth-related pathways (e.g., mTOR, EMT, and P2XR6) but positively regulates cell death-related pathways or tumor suppressors (e.g., P53, PTEN, and Notch1). When METTL3 is highly expressed, it positively regulates the NF-kB and SHH-GL1 pathways (proliferation-related pathways) and negatively regulates PTEN (Table 1).

Compared to METTL14, METTL3 seemly shows various expression patterns and affects different regulation pathways depending on the type of urological cancer, suggesting that METTL3 has organ-specific characteristics. Based on available data, modulation of m6A regulation may represent a new therapeutic target for urological cancer treatment. However, because of the limited number of available studies, we cannot fully elucidate the molecular mechanisms regulating the $\mathrm{m} 6 \mathrm{~A}$ modification in urological tumors. Additional studies are needed to thoroughly understand the mechanism and determine the therapeutic potential of targeting m6A regulation in urological tumors. Based on the existing results, METTL3 and METTL14 control cancer cell fate through cell growth- and cell death-related pathways. Although METTL3 and 14 have been a prominent focus of studies of m6A in urological tumors, the role of other enzymes is also worth studying. The m6A-related enzymes VIRMA and 
362 YTHDF3 have been implicated in testicular cancer. Additional research is needed to define the 363 mechanisms of these m6A enzymes in this disease.

364 Other researchers have studied the role of m6A-related genes in urological tumors from different

365

366

367

368

369

370

371

372

373

374

375

376

377

378

379

380

381

382

383

384

385

386

387

388

389

390

391

392

393

394

395

396

directions. Unlike our approach, they mainly focused on analyzing the expression of m6A-

related genes using the TCGA database and found that urological cancer tends to follow the same pattern, with the upregulation of methyltransferase related to higher tumor grade and stage. In addition, they looked not only at expression differences of 'Writers' in urological tumors but also differences in the expression of 'Erasers' and 'Readers' in these tumor types. Interestingly, VIRMA is upregulated in all four urological tumors, which indicates that it could be a potential molecular target worth exploring. Although these results are refreshing, the relevant conclusions and opinions must be confirmed experimentally (Lobo et al. 2018).

In this review, the potential molecular networks surrounding the m6A modification are described based on existing research. Although m6A has just emerged in urological oncology, it has already shown researchers a promising direction. The available data suggest that regulators of the m6A modification may represent new targets and biomarkers for the treatment and diagnosis or prognosis of urological cancers.

\section{Acknowledgements}

Thanks to Professor Han Fang, who serves for PTSD Laboratory of Department of Histology and Embryology in the Basic Medical College of China Medical University, for her strong guidance and support for this review, she has put forward valuable suggestions for the improvement of this paper.

\section{References}

Alarcon CR, Goodarzi H, Lee H, Liu X, Tavazoie S, and Tavazoie SF. 2015a. HNRNPA2B1 Is a Mediator of m(6)A-Dependent Nuclear RNA Processing Events. Cell 162:1299-1308. 10.1016/j.cell.2015.08.011

Alarcon CR, Lee H, Goodarzi H, Halberg N, and Tavazoie SF. 2015b. N6-methyladenosine marks primary microRNAs for processing. Nature 519:482-485. 10.1038/nature14281

Barros-Silva D, Lobo J, Guimaraes-Teixeira C, Carneiro I, Oliveira J, Martens-Uzunova ES, Henrique R, and Jeronimo C. 2020. VIRMA-Dependent N6-Methyladenosine Modifications Regulate the Expression of Long Non-Coding RNAs CCAT1 and CCAT2 in Prostate Cancer. Cancers (Basel) 12. 10.3390/cancers12040771

Boccaletto P, Machnicka MA, Purta E, Piatkowski P, Baginski B, Wirecki TK, de Crecy-Lagard V, Ross R, Limbach PA, Kotter A, Helm M, and Bujnicki JM. 2018. MODOMICS: a 
397

398

399

400

401

402

403

404

405

406

407

408

409

410

411

412

413

414

415

416

417

418

419

420

421

422

423

424

425

426

427

428

429

430

431

432

433

434

435

436

database of RNA modification pathways. 2017 update. Nucleic Acids Res 46:D303d307. 10.1093/nar/gkx1030

Bokar JA, Shambaugh ME, Polayes D, Matera AG, and Rottman FM. 1997. Purification and cDNA cloning of the AdoMet-binding subunit of the human mRNA (N6-adenosine)methyltransferase. Rna 3:1233-1247.

Bray F, Ferlay J, Soerjomataram I, Siegel RL, Torre LA, and Jemal A. 2018. Global cancer statistics 2018: GLOBOCAN estimates of incidence and mortality worldwide for 36 cancers in 185 countries. CA Cancer J Clin 68:394-424. 10.3322/caac.21492

Cai J, Yang F, Zhan H, Situ J, Li W, Mao Y, and Luo Y. 2019. RNA m(6)A Methyltransferase METTL3 Promotes The Growth Of Prostate Cancer By Regulating Hedgehog Pathway. Onco Targets Ther 12:9143-9152. 10.2147/ott.S226796

Center MM, Jemal A, Lortet-Tieulent J, Ward E, Ferlay J, Brawley O, and Bray F. 2012. International variation in prostate cancer incidence and mortality rates. Eur Urol 61:10791092. 10.1016/j.eururo.2012.02.054

Chadet S, Jelassi B, Wannous R, Angoulvant D, Chevalier S, Besson P, and Roger S. 2014. The activation of $\mathrm{P} 2 \mathrm{Y} 2$ receptors increases MCF-7 breast cancer cells migration through the MEK-ERK1/2 signalling pathway. Carcinogenesis 35:1238-1247. 10.1093/carcin/bgt493

Chen M, Nie ZY, Wen XH, Gao YH, Cao H, and Zhang SF. 2019a. m6A RNA methylation regulators can contribute to malignant progression and impact the prognosis of bladder cancer. Biosci Rep 39. 10.1042/bsr20192892

Chen M, Wei L, Law CT, Tsang FH, Shen J, Cheng CL, Tsang LH, Ho DW, Chiu DK, Lee JM, Wong CC, Ng IO, and Wong CM. 2018. RNA N6-methyladenosine methyltransferaselike 3 promotes liver cancer progression through YTHDF2-dependent posttranscriptional silencing of SOCS2. Hepatology 67:2254-2270. 10.1002/hep.29683

Chen XY, Zhang J, and Zhu JS. 2019b. The role of m(6)A RNA methylation in human cancer. Mol Cancer 18:103. 10.1186/s12943-019-1033-z

Cheng L, Albers P, Berney DM, Feldman DR, Daugaard G, Gilligan T, and Looijenga LHJ. 2018. Testicular cancer. Nat Rev Dis Primers 4:29. 10.1038/s41572-018-0029-0

Cheng M, Sheng L, Gao Q, Xiong Q, Zhang H, Wu M, Liang Y, Zhu F, Zhang Y, Zhang X, Yuan $\mathrm{Q}$, and Li Y. 2019. The m(6)A methyltransferase METTL3 promotes bladder cancer progression via AFF4/NF-kappaB/MYC signaling network. Oncogene 38:3667-3680. 10.1038/s41388-019-0683-z

Choe J, Lin S, Zhang W, Liu Q, Wang L, Ramirez-Moya J, Du P, Kim W, Tang S, Sliz P, Santisteban P, George RE, Richards WG, Wong KK, Locker N, Slack FJ, and Gregory RI. 2018. mRNA circularization by METTL3-elF3h enhances translation and promotes oncogenesis. Nature 561:556-560. 10.1038/s41586-018-0538-8

Cui Q, Shi H, Ye P, Li L, Qu Q, Sun G, Sun G, Lu Z, Huang Y, Yang CG, Riggs AD, He C, and Shi Y. 2017. m(6)A RNA Methylation Regulates the Self-Renewal and Tumorigenesis of Glioblastoma Stem Cells. Cell Rep 18:2622-2634. 10.1016/j.celrep.2017.02.059 
437 Deng X, Su R, Weng H, Huang H, Li Z, and Chen J. 2018. RNA N(6)-methyladenosine

438

439

440

441

442

443

444

445

446

447

448

449

450

451

452

453

454

455

456

457

458

459

460

461

462

463

464

465

466

467

468

469

470

471

472

473

474

475 modification in cancers: current status and perspectives. Cell Res 28:507-517. 10.1038/s41422-018-0034-6

Dominissini D, Moshitch-Moshkovitz S, Schwartz S, Salmon-Divon M, Ungar L, Osenberg S, Cesarkas K, Jacob-Hirsch J, Amariglio N, Kupiec M, Sorek R, and Rechavi G. 2012. Topology of the human and mouse m6A RNA methylomes revealed by m6A-seq. Nature 485:201-206. 10.1038/nature11112

Du Y, Hou G, Zhang H, Dou J, He J, Guo Y, Li L, Chen R, Wang Y, Deng R, Huang J, Jiang B, Xu M, Cheng J, Chen GQ, Zhao X, and Yu J. 2018. SUMOylation of the m6A-RNA methyltransferase METTL3 modulates its function. Nucleic Acids Res 46:5195-5208. 10.1093/nar/gky156

Gong D, Zhang J, Chen Y, Xu Y, Ma J, Hu G, Huang Y, Zheng J, Zhai W, and Xue W. 2019. The $\mathrm{m}(6) A$-suppressed P2RX6 activation promotes renal cancer cells migration and invasion through ATP-induced $\mathrm{Ca}(2+)$ influx modulating ERK1/2 phosphorylation and MMP9 signaling pathway. J Exp Clin Cancer Res 38:233. 10.1186/s13046-019-1223-y

Gu C, Wang Z, Zhou N, Li G, Kou Y, Luo Y, Wang Y, Yang J, and Tian F. 2019. Mettl14 inhibits bladder TIC self-renewal and bladder tumorigenesis through $\mathrm{N}(6)$-methyladenosine of Notch1. Mol Cancer 18:168. 10.1186/s12943-019-1084-1

Guo L, Lin M, Cheng Z, Chen Y, Huang Y, and Xu K. 2019. Identification of key genes and multiple molecular pathways of metastatic process in prostate cancer. PeerJ 7:e7899. 10.7717/peerj.7899

Han J, Wang JZ, Yang X, Yu H, Zhou R, Lu HC, Yuan WB, Lu JC, Zhou ZJ, Lu Q, Wei JF, and Yang H. 2019. METTL3 promote tumor proliferation of bladder cancer by accelerating pri-miR221/222 maturation in m6A-dependent manner. Mol Cancer 18:110. 10.1186/s12943-019-1036-9

Hao H, Wang Z, Ren S, Shen H, Xian H, Ge W, and Wang W. 2019. Reduced GRAMD1C expression correlates to poor prognosis and immune infiltrates in kidney renal clear cell carcinoma. PeerJ 7:e8205. 10.7717/peerj.8205

Jin H, Ying X, Que B, Wang X, Chao Y, Zhang H, Yuan Z, Qi D, Lin S, Min W, Yang M, and Ji W. 2019. N(6)-methyladenosine modification of ITGA6 mRNA promotes the development and progression of bladder cancer. EBioMedicine 47:195-207. 10.1016/j.ebiom.2019.07.068

Ke S, Pandya-Jones A, Saito Y, Fak JJ, Vagbo CB, Geula S, Hanna JH, Black DL, Darnell JE, Jr., and Darnell RB. 2017. m(6)A mRNA modifications are deposited in nascent premRNA and are not required for splicing but do specify cytoplasmic turnover. Genes Dev 31:990-1006. 10.1101/gad.301036.117

Leach RA, and Tuck MT. 2001. Expression of the mRNA (N6-adenosine)-methyltransferase Sadenosyl-L-methionine binding subunit mRNA in cultured cells. Int $\mathrm{J}$ Biochem Cell Biol 33:984-999. 10.1016/s1357-2725(01)00071-1 
476 Li E, Wei B, Wang X, and Kang R. 2020. METTL3 enhances cell adhesion through stabilizing

477

478

479

480

481

482

483

484

485

486

487

488

489

490

491

492

493

494

495

496

497

498

499

500

501

502

503

504

505

506

507

508

509

510

511

512

513

514 integrin beta1 mRNA via an m6A-HuR-dependent mechanism in prostatic carcinoma. Am J Cancer Res 10:1012-1025.

Li X, Tang J, Huang W, Wang F, Li P, Qin C, Qin Z, Zou Q, Wei J, Hua L, Yang H, and Wang Z. 2017a. The M6A methyltransferase METTL3: acting as a tumor suppressor in renal cell carcinoma. Oncotarget 8:96103-96116. 10.18632/oncotarget.21726

Li Z, Weng H, Su R, Weng X, Zuo Z, Li C, Huang H, Nachtergaele S, Dong L, Hu C, Qin X, Tang L, Wang Y, Hong GM, Huang H, Wang X, Chen P, Gurbuxani S, Arnovitz S, Li Y, Li S, Strong J, Neilly MB, Larson RA, Jiang X, Zhang P, Jin J, He C, and Chen J. 2017b. FTO Plays an Oncogenic Role in Acute Myeloid Leukemia as a N(6)-Methyladenosine RNA Demethylase. Cancer Cell 31:127-141. 10.1016/j.ccell.2016.11.017

Lin S, Choe J, Du P, Triboulet R, and Gregory RI. 2016. The m(6)A Methyltransferase METTL3 Promotes Translation in Human Cancer Cells. Mol Cell 62:335-345. 10.1016/j.molcel.2016.03.021

Liu J, Eckert MA, Harada BT, Liu SM, Lu Z, Yu K, Tienda SM, Chryplewicz A, Zhu AC, Yang Y, Huang JT, Chen SM, Xu ZG, Leng XH, Yu XC, Cao J, Zhang Z, Liu J, Lengyel E, and He C. 2018. m(6)A mRNA methylation regulates AKT activity to promote the proliferation and tumorigenicity of endometrial cancer. Nat Cell Biol 20:1074-1083. 10.1038/s41556018-0174-4

Liu J, Yue Y, Han D, Wang X, Fu Y, Zhang L, Jia G, Yu M, Lu Z, Deng X, Dai Q, Chen W, and He C. 2014. A METTL3-METTL14 complex mediates mammalian nuclear RNA N6adenosine methylation. Nat Chem Biol 10:93-95. 10.1038/nchembio.1432

Liu N, Dai Q, Zheng G, He C, Parisien M, and Pan T. 2015. N(6)-methyladenosine-dependent RNA structural switches regulate RNA-protein interactions. Nature 518:560-564. 10.1038/nature14234

Lobo J, Barros-Silva D, Henrique R, and Jeronimo C. 2018. The Emerging Role of Epitranscriptomics in Cancer: Focus on Urological Tumors. Genes (Basel) 9. 10.3390/genes9110552

Lobo J, Costa AL, Cantante M, Guimaraes R, Lopes P, Antunes L, Braga I, Oliveira J, Pelizzola M, Henrique R, and Jeronimo C. 2019. m(6)A RNA modification and its writer/reader VIRMA/YTHDF3 in testicular germ cell tumors: a role in seminoma phenotype maintenance. J Trans/ Med 17:79. 10.1186/s12967-019-1837-z

Ma JZ, Yang F, Zhou CC, Liu F, Yuan JH, Wang F, Wang TT, Xu QG, Zhou WP, and Sun SH. 2017. METTL14 suppresses the metastatic potential of hepatocellular carcinoma by modulating $\mathrm{N}(6)$-methyladenosine-dependent primary MicroRNA processing. Hepatology 65:529-543. 10.1002/hep.28885

Ma XX, Cao ZG, and Zhao SL. 2020. m6A methyltransferase METTL3 promotes the progression of prostate cancer via m6A-modified LEF1. Eur Rev Med Pharmacol Sci 24:3565-3571. 10.26355/eurrev_202004_20817 
515 Meyer KD, Patil DP, Zhou J, Zinoviev A, Skabkin MA, Elemento O, Pestova TV, Qian SB, and

516

517

518

519

520

521

522

523

524

525

526

527

528

529

530

531

532

533

534

535

536

537

538

539

540

541

542

543

544

545

546

547

548

549

550

551

552

553

554 Jaffrey SR. 2015. 5' UTR m(6)A Promotes Cap-Independent Translation. Cell 163:9991010. 10.1016/j.cell.2015.10.012

Moch H, Cubilla AL, Humphrey PA, Reuter VE, and Ulbright TM. 2016. The 2016 WHO Classification of Tumours of the Urinary System and Male Genital Organs-Part A: Renal, Penile, and Testicular Tumours. Eur Urol 70:93-105. 10.1016/j.eururo.2016.02.029

Nettersheim D, Berger D, Jostes S, Kristiansen G, Lochnit G, and Schorle H. 2019. N6Methyladenosine detected in RNA of testicular germ cell tumors is controlled by METTL3, ALKBH5, YTHDC1/F1/F2, and HNRNPC as writers, erasers, and readers. Andrology 7:498-506. 10.1111/andr.12612

North RA. 2002. Molecular physiology of P2X receptors. Physiol Rev 82:1013-1067. 10.1152/physrev.00015.2002

O'Reilly KE, Rojo F, She QB, Solit D, Mills GB, Smith D, Lane H, Hofmann F, Hicklin DJ, Ludwig DL, Baselga J, and Rosen N. 2006. mTOR inhibition induces upstream receptor tyrosine kinase signaling and activates Akt. Cancer Res 66:1500-1508. 10.1158/0008-5472.Can05-2925

Ping XL, Sun BF, Wang L, Xiao W, Yang X, Wang WJ, Adhikari S, Shi Y, Lv Y, Chen YS, Zhao X, Li A, Yang Y, Dahal U, Lou XM, Liu X, Huang J, Yuan WP, Zhu XF, Cheng T, Zhao YL, Wang X, Rendtlew Danielsen JM, Liu F, and Yang YG. 2014. Mammalian WTAP is a regulatory subunit of the RNA N6-methyladenosine methyltransferase. Cell Res 24:177189. $10.1038 / \mathrm{cr} .2014 .3$

Roobol MJ, and Carlsson SV. 2013. Risk stratification in prostate cancer screening. Nat Rev Urol 10:38-48. 10.1038/nrurol.2012.225

Sanli O, Dobruch J, Knowles MA, Burger M, Alemozaffar M, Nielsen ME, and Lotan Y. 2017. Bladder cancer. Nat Rev Dis Primers 3:17022. 10.1038/nrdp.2017.22

Scholler E, Weichmann F, Treiber T, Ringle S, Treiber N, Flatley A, Feederle R, Bruckmann A, and Meister G. 2018. Interactions, localization, and phosphorylation of the $\mathrm{m}(6) \mathrm{A}$ generating METTL3-METTL14-WTAP complex. Rna 24:499-512. 10.1261/rna.064063.117

Shaw RJ, and Cantley LC. 2006. Ras, PI(3)K and mTOR signalling controls tumour cell growth. Nature 441:424-430. 10.1038/nature04869

Shen MM, and Abate-Shen C. 2010. Molecular genetics of prostate cancer: new prospects for old challenges. Genes Dev 24:1967-2000. 10.1101/gad.1965810

Sledz P, and Jinek M. 2016. Structural insights into the molecular mechanism of the $\mathrm{m}(6) A$ writer complex. Elife 5. 10.7554/eLife.18434

Visvanathan A, Patil V, Arora A, Hegde AS, Arivazhagan A, Santosh V, and Somasundaram K. 2018. Essential role of METTL3-mediated $\mathrm{m}(6) \mathrm{A}$ modification in glioma stem-like cells maintenance and radioresistance. Oncogene 37:522-533. 10.1038/onc.2017.351

Vu LP, Cheng Y, and Kharas MG. 2019. The Biology of m(6)A RNA Methylation in Normal and Malignant Hematopoiesis. Cancer Discov 9:25-33. 10.1158/2159-8290.Cd-18-0959 
555

556

557

558

559

560

561

562

563

564

565

566

567

568

569

570

571

572

573

574

575

576

577

578

579

580

581

582

583

584

585

586

587

588

589

590

591

592

593

594

595

Vu LP, Pickering BF, Cheng Y, Zaccara S, Nguyen D, Minuesa G, Chou T, Chow A, Saletore Y, MacKay M, Schulman J, Famulare C, Patel M, Klimek VM, Garrett-Bakelman FE, Melnick A, Carroll M, Mason CE, Jaffrey SR, and Kharas MG. 2017. The N(6)methyladenosine $(\mathrm{m}(6) \mathrm{A})$-forming enzyme METTL3 controls myeloid differentiation of normal hematopoietic and leukemia cells. Nat Med 23:1369-1376. 10.1038/nm.4416

Wang P, Doxtader KA, and Nam Y. 2016a. Structural Basis for Cooperative Function of Mettl3 and Mettl14 Methyltransferases. Mol Cell 63:306-317. 10.1016/j.molcel.2016.05.041

Wang Q, Zhang H, Chen Q, Wan Z, Gao X, and Qian W. 2019. Identification of METTL14 in Kidney Renal Clear Cell Carcinoma Using Bioinformatics Analysis. Dis Markers 2019:5648783. 10.1155/2019/5648783

Wang X, Feng J, Xue Y, Guan Z, Zhang D, Liu Z, Gong Z, Wang Q, Huang J, Tang C, Zou T, and Yin P. 2016b. Structural basis of N(6)-adenosine methylation by the METTL3METTL14 complex. Nature 534:575-578. 10.1038/nature18298

Wang X, Huang J, Zou T, and Yin P. 2017. Human m(6)A writers: Two subunits, 2 roles. RNA Biol 14:300-304. 10.1080/15476286.2017.1282025

Wang X, Lu Z, Gomez A, Hon GC, Yue Y, Han D, Fu Y, Parisien M, Dai Q, Jia G, Ren B, Pan T, and $\mathrm{He}$ C. 2014. N6-methyladenosine-dependent regulation of messenger RNA stability. Nature 505:117-120. 10.1038/nature12730

Weng H, Huang H, Wu H, Qin X, Zhao BS, Dong L, Shi H, Skibbe J, Shen C, Hu C, Sheng Y, Wang Y, Wunderlich M, Zhang B, Dore LC, Su R, Deng X, Ferchen K, Li C, Sun M, Lu Z, Jiang X, Marcucci G, Mulloy JC, Yang J, Qian Z, Wei M, He C, and Chen J. 2018. METTL14 Inhibits Hematopoietic Stem/Progenitor Differentiation and Promotes Leukemogenesis via mRNA m(6)A Modification. Cell Stem Cell 22:191-205.e199. 10.1016/j.stem.2017.11.016

Wu G, Wang F, Li K, Li S, Zhao C, Fan C, and Wang J. 2019. Significance of TP53 mutation in bladder cancer disease progression and drug selection. PeerJ 7:e8261. 10.7717/peerj.8261

Xiang Y, Laurent B, Hsu CH, Nachtergaele S, Lu Z, Sheng W, Xu C, Chen H, Ouyang J, Wang S, Ling D, Hsu PH, Zou L, Jambhekar A, He C, and Shi Y. 2017. RNA m(6)A methylation regulates the ultraviolet-induced DNA damage response. Nature 543:573-576. 10.1038/nature21671

Xianyong L, Runyun G, Kun-bin K, Peng G, Tonghai L, Qingpeng C, Yin C, and Hui Z. 2019. Expression and clinical significance of METTL3 in prostate cancer. The Journal of Practical Medicine 35:75-79.

Xie H, Li J, Ying Y, Yan H, Jin K, Ma X, He L, Xu X, Liu B, Wang X, Zheng X, and Xie L. 2020. METTL3/YTHDF2 $\mathrm{m}(6) \mathrm{A}$ axis promotes tumorigenesis by degrading SETD7 and KLF4 mRNAs in bladder cancer. J Cell Mol Med 24:4092-4104. 10.1111/jcmm.15063

Yang F, Jin H, Que B, Chao Y, Zhang H, Ying X, Zhou Z, Yuan Z, Su J, Wu B, Zhang W, Qi D, Chen D, Min W, Lin S, and Ji W. 2019. Dynamic m(6)A mRNA methylation reveals the role of METTL3-m(6)A-CDCP1 signaling axis in chemical carcinogenesis. Oncogene 38:4755-4772. 10.1038/s41388-019-0755-0

Peer] reviewing PDF | (2020:03:46959:2:0:NEW 26 Jun 2020) 
596

597

598

599

600

601

602

603

604

605

606

607

608

609

610

611

612

613

614

615

616

617

618

619

620

621

Zhang C, Samanta D, Lu H, Bullen JW, Zhang H, Chen I, He X, and Semenza GL. 2016.

Hypoxia induces the breast cancer stem cell phenotype by HIF-dependent and ALKBH5mediated m(6)A-demethylation of NANOG mRNA. Proc Natl Acad Sci U S A 113:E20472056. 10.1073/pnas.1602883113

Zhang S, Zhao BS, Zhou A, Lin K, Zheng S, Lu Z, Chen Y, Sulman EP, Xie K, Bogler O, Majumder S, He C, and Huang S. 2017. m(6)A Demethylase ALKBH5 Maintains Tumorigenicity of Glioblastoma Stem-like Cells by Sustaining FOXM1 Expression and Cell Proliferation Program. Cancer Cell 31:591-606.e596. 10.1016/j.ccell.2017.02.013

Zhao S, Liu J, Nanga P, Liu Y, Cicek AE, Knoblauch N, He C, Stephens M, and He X. 2019. Detailed modeling of positive selection improves detection of cancer driver genes. Nat Commun 10:3399. 10.1038/s41467-019-11284-9

Zheng W, Dong X, Zhao Y, Wang S, Jiang H, Zhang M, Zheng X, and Gu M. 2019. Multiple Functions and Mechanisms Underlying the Role of METTL3 in Human Cancers. Front Oncol 9:1403. 10.3389/fonc.2019.01403

Zhong X, Yu J, Frazier K, Weng X, Li Y, Cham CM, Dolan K, Zhu X, Hubert N, Tao Y, Lin F, Martinez-Guryn K, Huang Y, Wang T, Liu J, He C, Chang EB, and Leone V. 2018. Circadian Clock Regulation of Hepatic Lipid Metabolism by Modulation of m(6)A mRNA Methylation. Cell Rep 25:1816-1828.e1814. 10.1016/j.celrep.2018.10.068

Zhou J, Wang J, Hong B, Ma K, Xie H, Li L, Zhang K, Zhou B, Cai L, and Gong K. 2019. Gene signatures and prognostic values of $\mathrm{m} 6 \mathrm{~A}$ regulators in clear cell renal cell carcinoma - a retrospective study using TCGA database. Aging (Albany NY) 11:1633-1647. 10.18632/aging.101856

Zhu F, Qian W, Zhang H, Liang Y, Wu M, Zhang Y, Zhang X, Gao Q, and Li Y. 2017. SOX2 Is a Marker for Stem-like Tumor Cells in Bladder Cancer. Stem Cell Reports 9:429-437. 10.1016/j.stemcr.2017.07.004 


\section{Figure 1}

METTL3 and METTL14 regulatory network in kidney cancer

The red arrows in the figure represent the promoting effect, and the yellow arrows represent the inhibiting effect. PI3K / AKT / mTOR, EMT and P2RX6 play an oncogenic role in kidney cancer, while p53 and PTEN play a tumor suppressive role. METTL3 and METTL14 play a role in suppressing kidney cancer by inhibiting or promoting some pathways, respectively. At the same time, they also accept regulation from upstream molecules or pathways. 


\section{METTL3/14 regulatory network in kidney cancer.}

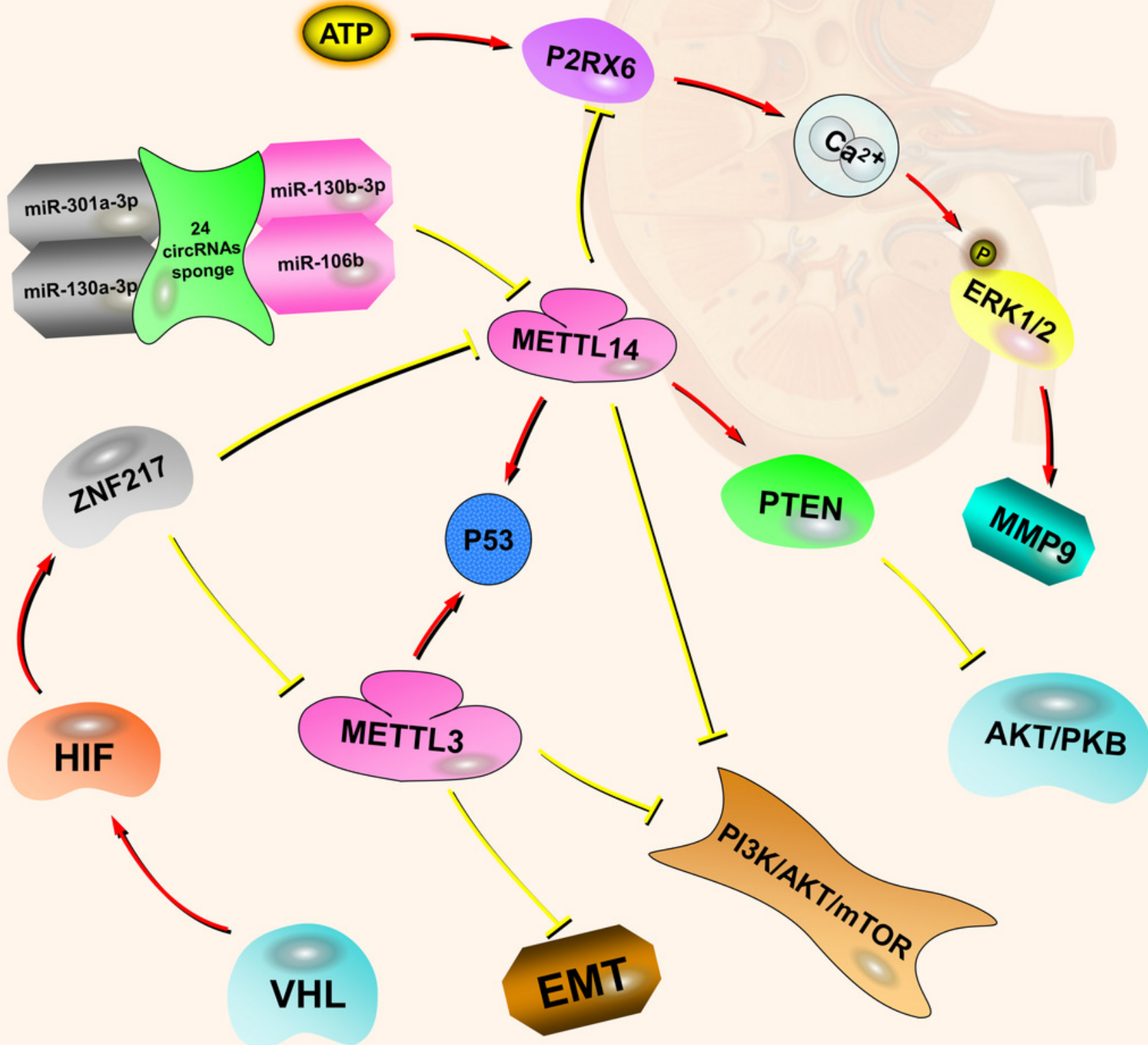




\section{Figure 2}

METTL3 and METTL14 regulatory network in bladder cancer

The red arrows in the figure represent the promoting effect, and the yellow arrows represent the inhibiting effect. AFF4 /NF-KB/ MYC, DGCR8-pri-miR221/222-PTEN, CDCP1, ITGA6 and Notch1 play oncogenic role in bladder cancer, while SETD7 and KLF4 play a tumor suppressive role. METTL3 can play an oncogenic role in bladder cancer through a variety of pathways. METTL14 is the opposite, although there are not many related studies, it is certain that it plays a tumor suppressive role in bladder cancer. 
METTL3/14 regulatory network in bladder cancer.

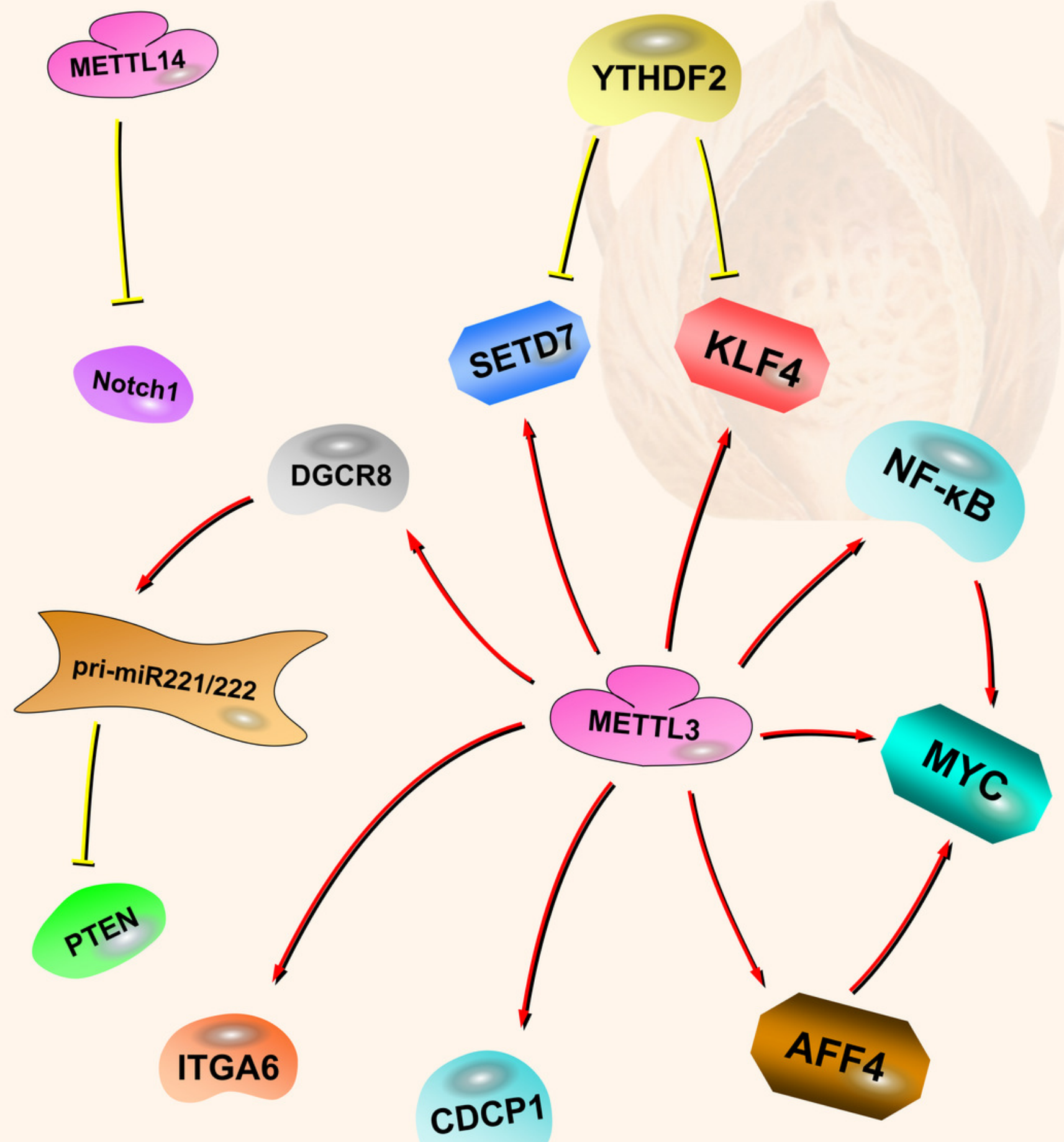




\section{Figure 3}

METTL3 regulatory network in prostate cancer

The red arrows in the figure represent the promoting effect. There are not many researches on METTL3 in prostate cancer compared with kidney cancer and bladder cancer. It is certain that it plays a role in oncogenesis by affecting downstream pathway SHH-GLII, ITGB1, and LEF1-Wnt / $\beta$-catenin. At present, there is no research on the pathway of METTL14 in prostate cancer. 
METTL3 regulatory network in prostate cancer.

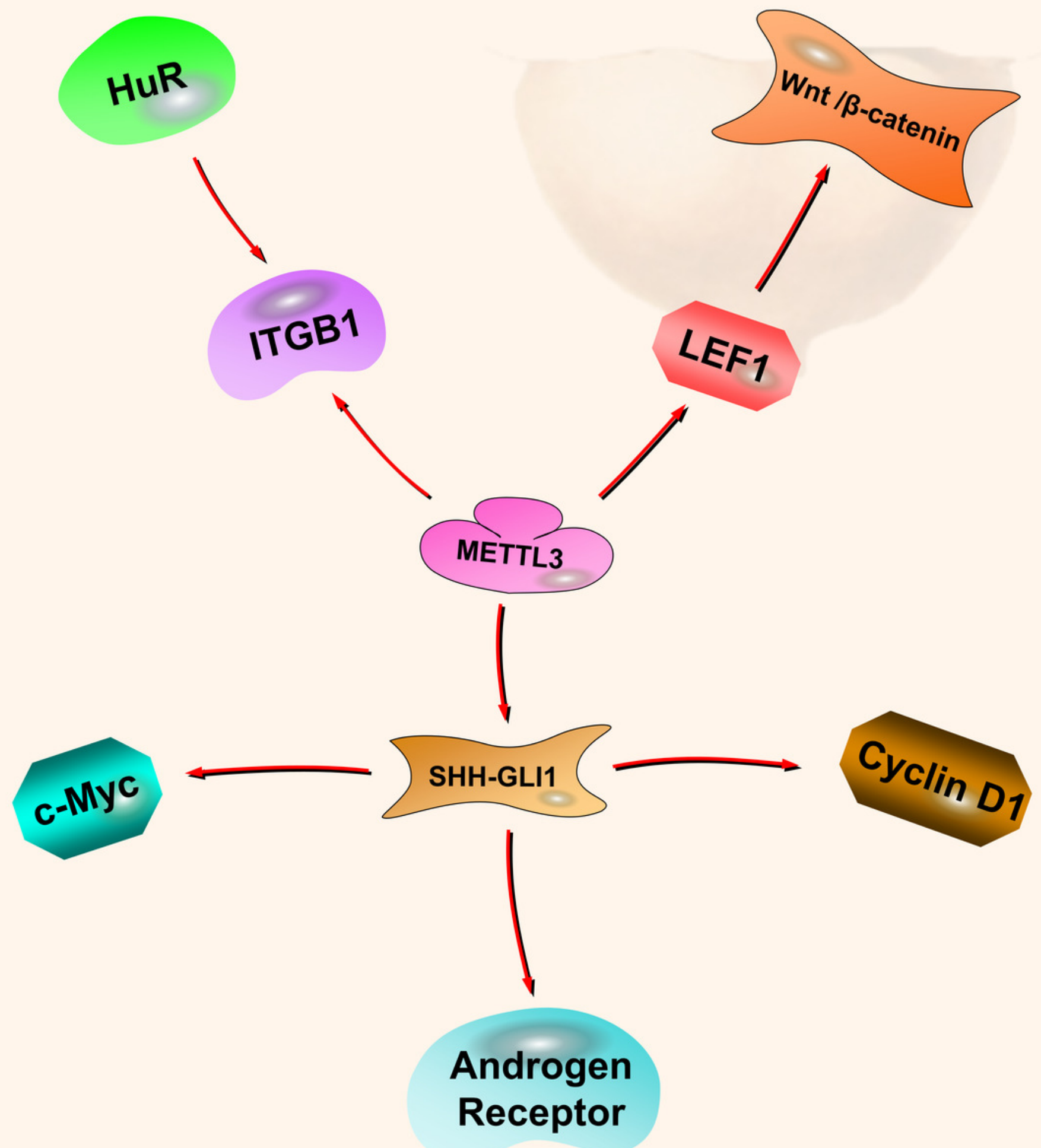




\section{Table $\mathbf{1}$ (on next page)}

Summary of METTL3 and METTL14 related pathways in urological tumors 


\begin{tabular}{|c|c|c|c|c|c|c|}
\hline Diseases & Component & Role & Source of experimental evidence & Regulation & Potential signal pathway & Author \& Refs \\
\hline \multirow[b]{3}{*}{$\begin{array}{l}\text { Kidney } \\
\text { cancer }\end{array}$} & METTL3 & $\begin{array}{l}\text { Tumor } \\
\text { suppressor }\end{array}$ & $\begin{array}{l}\text { RCC and matched histologically-normal } \\
\text { renal tissues are from } 145 \text { RCC patients; } \\
\text { RCC cell lines (CAKI-1, CAKI-2 and } \\
\text { ACHN); a normal renal tubular epithelial } \\
\text { cell line (HK-2); BALB/c nude mice }\end{array}$ & Down-regulation & $\begin{array}{l}\text { METTL3-PI3K/AKT/mTOR } \\
\text { METTL3-EMT }\end{array}$ & (Li et al. 2017) \\
\hline & METTL3 & $\begin{array}{l}\text { Tumor } \\
\text { suppressor }\end{array}$ & $\begin{array}{l}528 \text { ccRCC patients with CNV data and } \\
\text { pathology reports from the TCGA } \\
\text { database; GSEA database }\end{array}$ & Up-regulation & $\begin{array}{l}\text { VHL-HIF-ZNF217-METTL3- } \\
\text { PI3K/AKT/mTOR } \\
\text { VHL-HIF-ZNF217-METTL3-p53 }\end{array}$ & (Zhou et al. 2019) \\
\hline & METTL14 & $\begin{array}{l}\text { Tumor } \\
\text { suppressor }\end{array}$ & $\begin{array}{l}\text { Online } \square \text { databases } \\
\text { (TCGAportal,GTExPortal,UALCAN,GEPI } \\
\text { A2,MEXPRESS,RMBasev2.0,OncoLnc,sta } \\
\text { rBase,circBank,STRING... ) } \\
17 \text { groups of renal cell carcinoma tissues } \\
\text { and adjacent tissues received in patients } \\
\text { with partial or complete kidney resection; } \\
\text { Renal cancer cell line(OS-RC-2,786- } \\
\text { O,HEK-293, SN12-PM6, SW839, } \\
\text { A498); Human cortical proximal tubule } \\
\text { epithelial cell line (HK-2); Nude mice; } \\
\text { Online databases (TCGA, UALCAN...) }\end{array}$ & Down-regulation & $\begin{array}{l}\text { VHL-HIF-ZNF217-METTL14- } \\
\text { PI3K/AKT/mTOR } \\
\text { VHL-HIF-ZNF217-METTL14- } \\
\text { p53 } \\
\text { circRNAs-miRNAs-METTL14- } \\
\text { PTEN-AKT/PKB }\end{array}$ & (Gong et al. 2019) \\
\hline & METTL3 & Oncogene & $\begin{array}{l}\text { Human/mouse bladder cancer samples; } \\
\text { bladder cancer cell lines (5637, UM-UC-3); } \\
\text { Immortalized epithelial cells (SV-HUC-1); } \\
\text { GSEA database }\end{array}$ & Up-regulation & METTL3-AFF4 /NF-кB/ MYC & (Cheng et al. 2019) \\
\hline & METTL3 & Oncogene & $\begin{array}{l}\text { Human/mouse bladder cancer samples; } \\
\text { bladder cancer cell lines (EJ, T24) }\end{array}$ & Up-regulation & $\begin{array}{l}\text { METTL3-DGCR8-pri- } \\
\text { miR221/222-PTEN }\end{array}$ & (Han et al. 2019) \\
\hline $\begin{array}{l}\text { Bladder } \\
\text { cancer }\end{array}$ & METTL3 & Oncogene & $\begin{array}{l}\text { Formalin-fixed paraffin-embedded (FFPE) } \\
\text { tissue from } 114 \text { cases of bladder cancer and } \\
30 \text { cases of cystitis with radical cystectomy } \\
\text { and bladder biopsy; Human prostate }\end{array}$ & Up-regulation & METTL3 -CDCP1 & (Yang et al. 2019) \\
\hline
\end{tabular}




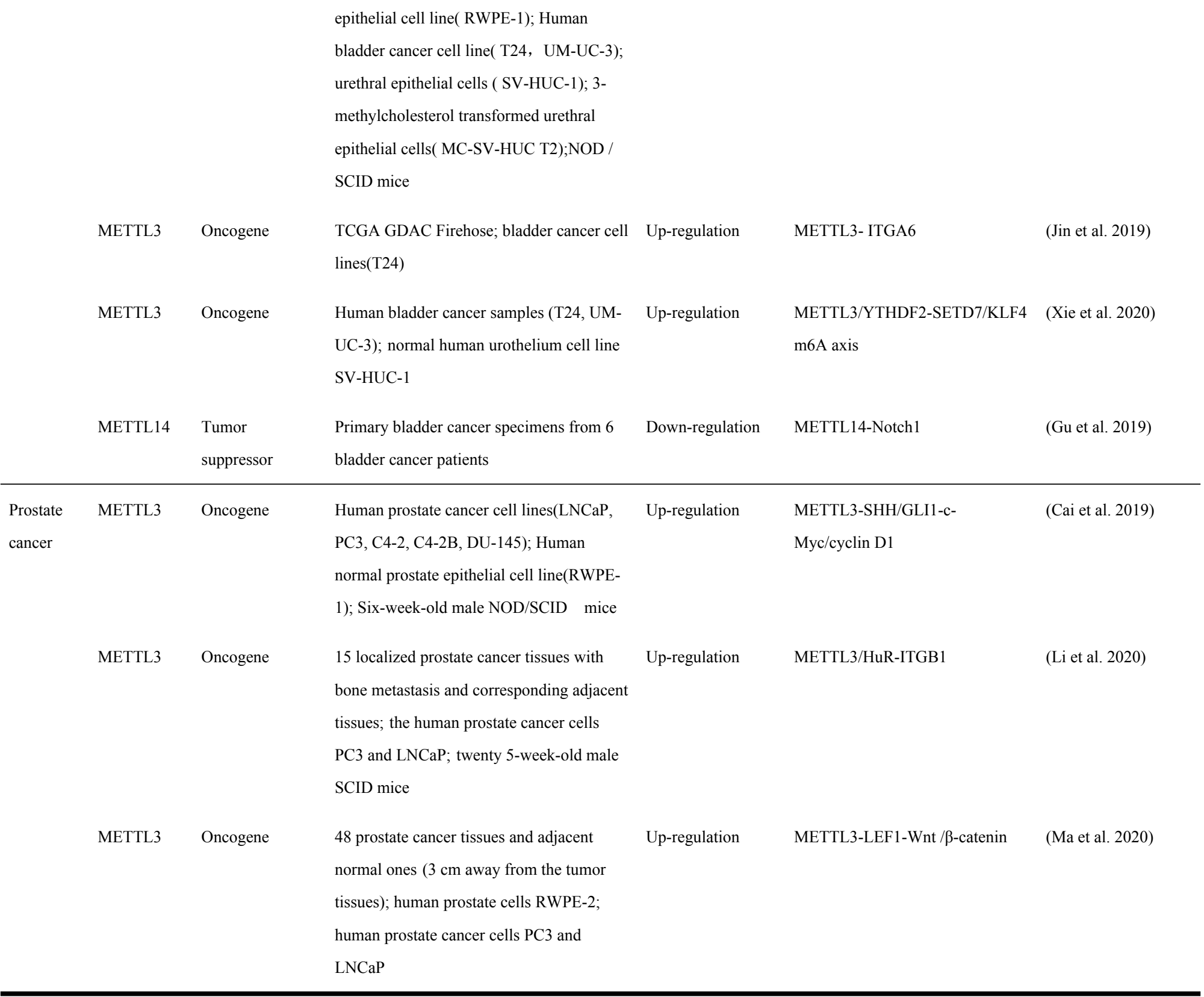

